\title{
COUNTING REAL $J$-HOLOMORPHIC DISCS AND SPHERES IN DIMENSION FOUR AND SIX
}

\author{
CHEOL-HYUN CHO
}

\begin{abstract}
We provide another proof that the signed count of the real $J$ holomorphic spheres (or $J$-holomorphic discs) passing through a generic real configuration of $k$ points is independent of the choice of the real configuration and the choice of $J$, if the dimension of the Lagrangian submanifold $L$ (fixed point set of involution) is two or three, and also if we assume $L$ is orientable and relatively spin. We also assume that $M$ is strongly semi-positive. This theorem was first proved by Welschinger in a more general setting, and we provide more natural approach using the signed degree of an evaluation map.
\end{abstract}

\section{Introduction}

Let $(M, \omega)$ be a symplectic manifold with an anti-symplectic involution $\tau$ whose fixed point set is a Lagrangian submanifold $L$. We choose a generic compatible almost complex structure $J$, which satisfies $(\tau)_{*} J=-J$. We assume that $M, L$ and $J$ satisfies the above condition throughout the paper. Additionally, we assume that $L$ is orientable and (relatively) spin, and $M$ is strongly semi-positive, which means that for every spherical class $\alpha \in H_{2}(M, \mathbb{Z})$ such that $[\omega](\alpha)>0$, the inequality $c_{1}(M) \alpha \geq 2-n$ implies $c_{1}(M) \geq 1$. The first condition implies that the moduli spaces of $J$-holomorphic discs are orientable. The second condition implies that the moduli space of simple real $J$-holomorphic (or $J_{t}$-holomorphic) discs form pseudo-cycle modulo disc bubbling (see Section 6 for definition).

In this paper, we study the counting of $J$-holomorphic discs $w:\left(D^{2}, \partial D^{2}\right) \rightarrow$ $(M, L)$ with the above assumptions or real $J$-holomorphic spheres

$$
u_{\mathbb{C}}:\left(\mathbb{C} P^{1}, \operatorname{con} j\right) \rightarrow(M, \tau)
$$

preserving real structures. We call them real $J$-holomorphic discs (or spheres) from now on.

Received February 14, 2007.

2000 Mathematics Subject Classification. 54D45, 14 N35.

Key words and phrases. holomorphic dises, anti-symplectic involution, Welschinger invariants. 
Theorem. Assume $M, \omega, L, J$ satisfy the above assumptions. If the dimension of $L$ is two or three, then there is a signed count of $J$-holomorphic spheres (and discs) which passes through a given real configuration of points such that it is independent of the choice of a real configuration of points or the choice of a generic almost complex structure.

This result was proved by Welschinger in his beautiful but mysterious papers [10], [11], [12] in a more general setting. The purpose of this note is to provide conceptually more natural approach to the proof of the invariance of the counting using the moduli spaces of $J$-holomorphic discs.

First of all, one naturally expect that the count of the real $J$-holomorphic spheres should be related to the counting of real $J$-holomorphic discs since the former can be obtained as a complex double of the latter. But the exact statement with orientation is rather complicated.

The most natural way to define an intersection number is as a degree of a certain map $f: M \rightarrow N$ where $M, N$ are oriented manifolds (for $N$ connected). From the basic transversality theory, the signed degree of $f, f^{-1}(n)$ for some $n \in N$, is well-defined, and invariant under the choice of $n \in N$ if the manifold $M$ has no boundary. In our case, we may try to consider an evaluation map $e v_{k}$ from the moduli space of real $J$-holomorphic spheres to $L^{k}$ and consider its signed degree. But the moduli spaces of real $J$-holomorphic spheres are not known to be orientable (in fact, the Proposition 2.1 suggest that it is not orientable in general). Hence we can not define the count as a degree of the map $e v_{k}$ from the moduli space of real holomorphic spheres.

Our approach is to use the moduli spaces of $J$-holomorphic discs instead, because they carry coherent orientations when the Lagrangian submanifold $L$ is orientable and (relatively) spin. But the disc moduli spaces have boundaries (actually corners) provided by the splitting of domain into two (or more) disc (or sphere) components. Therefore, the signed degree of $e v_{k}$ for each homotopy class of the discs is not well-defined. The idea, then is to put together several moduli spaces of holomorphic discs to make codimension one boundaries cancel out each other, so that the sum of signed degree is well-defined. We use the conjugation $\tau$ to make sure that there is always a matching pair for each boundary term. But, this also does not work, as the orientation changes of the conjugations depend on the Maslov indices of the holomorphic discs and the number of marked points. Hence, as we put together these moduli spaces of holomorphic discs, some boundaries cancel out but some of them do not cancel out.

But we show that for $\operatorname{dim}(L)=3$, the images of the boundary strata (under the suitable evaluation maps) always cancel out if the image is of codimension one. If the evaluation images are of higher codimension, they are irrelevant to the proof of the well-definedness of the degree of the evaluation map. Hence, in this case, the signed degree is well-defined. 
For the case $\operatorname{dim}(L)=2$, we need to add an additional sign corresponding to the cyclic ordering of the boundary marked points, and we show that the modified counting (which we call $B$-count) is well-defined as in the case of $\operatorname{dim}(L)=3$.

The approach taken by Welschinger is to define a sign (spinor state) to each preimage curve, (not the whole moduli space), and to prove the invariance of the signed count as the configuration of points changes. Configurations might pass though critical points of the evaluation map $e v_{k}$ or a reducible curve. (The critical points of the evaluation maps are such as the images of cuspidal curves [5]). Hence his proof is quite analytical as he needs to analyze the change of spinor state as the configuration crosses the critical values of the evaluation maps.

Our proof is rather combinatorial as we focus on the cancellation of signs. We can bypass the analysis on the transversality of the evaluation maps, as the well-definedness of the degree can be proved without such analysis.

Our method does not generalize to the higher dimensional cases (see [12]). For $\operatorname{dim}(L) \geq 4$, the counts we define in this paper, may changes as the configuration of points passes through reducible curves in general. This is because there may be too many types of reducible curves, and it is not possible to guarantee the cancellation of all codimension one images.

When this work was made available online at the arXiv [2], we learned of the similar work in progress by Jake Solomon whose work appeared online after two monthes [8]. He has discussed the case when the Lagrangian submanifold has dimension two and unorientable, and proved that the count of pseudoholomorphic dises agrees with Welschinger invariants.

Acknowledgement. We would like to thank Seongchun Kwon for reading the draft version and for the helpful suggestions. We would like to thank JeanYves Welschinger for explaining his results during his visit to Northwestern University in 2004.

\section{Orientation of the moduli space of discs and its conjugates}

\subsection{Orientation and conjugation.}

Suppose $M, \omega, J, L, \tau$ satisfy the assumption given in the introduction. Recall that for any $J$-holomorphic map $w:\left(D^{2}, \partial D^{2}\right) \mapsto(M, L)$, its conjugate $J$-holomorphic map is defined as

$$
\widetilde{w}(z)=\tau \circ w(\bar{z}) \text { for } z \in D^{2} .
$$

We denote the homology classes as $\beta=[w] \in H_{2}(M, L)$, and $\tau_{*}(\beta)=[\widetilde{w}]$. Let us first recall the following proposition from [3], which analyzed the orientation change under the conjugation. The space of holomorphic discs were shown to be orientable in [3] when $L$ is orientable and relatively spin. 
Proposition 2.1 ([3] Proposition 11.5, Corollary 11.9). The map

$$
\tau_{*}: \mathcal{M}_{0, k}(\beta) \rightarrow \mathcal{M}_{0, k}\left(\tau_{*}(\beta)\right)
$$

given by

$$
\left(w, z_{0}, \ldots, z_{k-1}\right) \mapsto\left(\widetilde{w}, \bar{z}_{0}, \ldots, \bar{z}_{k-1}\right)
$$

is orientation preserving if and only if

$$
\mu_{L}(\beta)+2 k \equiv 0 \quad(\bmod 4) .
$$

Remark 2.1. The sign of $\mathcal{M}_{0, k}(\beta)$ in this proposition does not incorporate the cyclic ordering factor, which we will explain later. For the definition of the moduli spaces used in this paper, see Section 6 .

Note that we have the following commuting diagram for each $k$,

$$
\begin{array}{ccc}
\mathcal{M}_{0, k}(\beta) & \stackrel{e v_{k, \beta}}{\longrightarrow} & L^{k} \\
\tau_{*} \downarrow & & \| \\
\mathcal{M}_{0, k}\left(\tau_{*}(\beta)\right) & \stackrel{e v_{k, \tau_{*} \beta}}{\longrightarrow} & L^{k} .
\end{array}
$$

Here $e v_{k, \beta}: \mathcal{M}_{0, k}(\beta) \mapsto L^{k}$ is the evaluation map at the boundary marked points. Therefore, the evaluation images exactly equal to each other and the difference is the orientations of the domain moduli spaces, which can be compared using the above theorem. The rought idea of the proof is as follows (see [3] for the exact details.) Note that the space of holomorphic discs of homotopy class $\beta$ has real (virtual) dimension $n+\mu(\beta)$, where its $n$-dimensional part of the tangent space of the moduli space is oriented by spin structure of $L$, and the remaining part of dimension $\mu(\beta)$ carry a complex orientation. Then, note that the conjugation $\mathbb{C}^{\mu / 2} \rightarrow \mathbb{C}^{\mu / 2}$ is orientation preserving if and only if $\mu \equiv 4$ $(\bmod 4)$, and the conjugation $\left(\partial D^{2}\right)^{k} \rightarrow\left(\partial D^{2}\right)^{k}$ is orientation preserving if and only if $k$ is even.

A good example of this is the case of $\left(\mathbb{C} P^{1}, \mathbb{R} P^{1}\right)$. Denote the upperhemisphere disc by $U$ and the lower-hemisphere disc by $L$ (their Maslov indices are two). In [1], we show that for the obstruction cycle (the case $k=1$, hence $\mu+2 k=4)$, the contributions of $U$ and $L$ does not cancel out, and for the boundary map of Floer cohomology (the case $k=2$, hence $\mu+2 k=6$ ), the contributions cancel out.

Another example is the case of special Lagrangian submanifolds in CalabiYau 3-folds which is given by anti-symplectic involution. In this case, virtual dimension of the moduli space of holomorphic disc is zero and we can count such holomorphic discs. First note that the sign of conjugation in this case with one marked point is always negative, because Maslov index vanishes. Hence whenever there is a disc bubbling (he bubble component is attached to the main component with one marked point), the above sign comparison shows that they occur in pairs with opposite sign which cancels out as explained in this paper. Yet the count is not an invariant in general as announced by Kenji Fukaya in his talk at Northwestern University and Waterloo in 2004. In this case, he has 
pointed out that there is an additional codimension one phenomenon, which is the sphere bubbling where the remaining disc component becomes a constant disc. Solomon [8] has shown this explicitly using the work of Fukaya, Oh, Ohta and Ono [3], where he restricts the homology class of the holomorphic disc not to be spherical so that such a phenomenon cannot happen.

\subsection{Cyclic ordering of marked points: $A$ and $B$ counts}

Consider the moduli space of distinct $k$ marked points on $\partial D^{2}$, denoted as $\mathcal{M}_{0, k}^{b}$. Here we assume $k \geq 3$. Here the marked points can be considered as a subset of $\left(\partial D^{2}\right)^{k} \backslash \Delta$ modulo $P S L(2: \mathbb{R})$ action where

$$
\Delta:=\left\{\left(z_{0}, \ldots, z_{k-1}\right) \in\left(\partial D^{2}\right)^{k} \mid z_{i}=z_{j} \text { for some } i \neq j\right\} .
$$

It consists of $(k-1)$ ! connected components according to the cyclic ordering of the marked points on $S^{1}$, therefore the moduli space of $J$-holomorphic discs $M_{0, k}(\beta)$ also has at least $(k-1)$ ! connected components.

As they are disconnected, there are two ways to orient the whole moduli spaces $\mathcal{M}_{0, k}(\beta)$. One ways is to orient the marked points as a subset of $\left(\partial D^{2}\right)^{k}$ with counterclockwise orientation on $\partial D^{2}$ (modulo $P S L(2, \mathbb{R})$ ).

The other way is to incorporate a factor regarding the cyclic ordering of the marked points. Namely, in the latter case, orientation may be defined by the following relation: For a permutation $\sigma$ of $\{1, \ldots, k\}$ (for $k \geq 3$ ), the map $\sigma_{*}: \mathcal{M}_{0, k}(\beta) \rightarrow \mathcal{M}_{0, k}(\beta)$ defined by

$$
\sigma_{*}\left(w,\left(z_{1}, \ldots, z_{k}\right)\right)=\left(w,\left(z_{\sigma(1)}, \ldots, z_{\sigma(k)}\right)\right)
$$

is orientation preserving if and only if $\sigma$ is an even permutation.

This provides two different orientations on the moduli spaces $\mathcal{M}_{0, k}(\beta)$. Therefore, if we consider the count based on each orientation, we get two different signed counts of $J$-holomorphic discs. We will call the count, $A$-count (resp. $B$-count) if the orientation does not (resp. does) incorporate cyclic ordering factor. We remark that the cyclic ordering factor was used to in [3] in the construction of $A_{\infty}$-algebra of Lagrangian submanifolds, but it was not used in a crucial way as $A_{\infty}$ structure is constructed using the main component.

For the purpose of the $B$-count, it is sometimes helpful to consider each connected pieces of the moduli space $\mathcal{M}_{0, k}(\beta)$ separately. Hence, we will denote by $\mathcal{M}_{0, k}^{\text {main }}(\beta)$ the connected component where the marked point are cyclically ordered counter clockwise as $\left(z_{0}, \ldots, z_{k-1}\right)$, and by $\mathcal{M}_{0, k}^{\sigma(\operatorname{main})}(\beta)$ if the marked points $\left(z_{\sigma(0)}, \ldots, z_{\sigma(k-1)}\right)$ are cyclically ordered for a permutation $\sigma$ of the index set $\{0, \ldots, k-1\}$.

\subsection{Comparison of signs of the discs and its conjugate in the count- ing}

For any $J$-holomorphic disc $w$ passing through a given configuration of real points, the conjugate disc $\widetilde{w}$ also passes through the same configuration of 
points. We compare the sign of intersections for $w$ and $\widetilde{w}$ as we consider counting problems.

Suppose the number of $J$-holomorphic discs of Maslov index $\mu$ passing through $k$ points on the real Lagrangian submanifold is finite (for a regular $J$ as chosen in Section 6). Namely, we will assume that

$$
n+\mu(\beta)-3+k(1-n)=0 .
$$

Suppose the disc $\left(w,\left(z_{0}, \ldots, z_{k-1}\right)\right)$ intersects the configuration of $k$ real points $\left\{x_{0}, \ldots, x_{k-1}\right\}$ at the corresponding marked points. The case of real configuration will be carried out in Section 5 .

We define the orientation of the intersection to be the usual preimage orientation of

$$
e v_{k, \beta}^{-1}\left(x_{1} \times \cdots \times x_{k-1}\right)
$$

(see [4] Chapter 3 for the standard definition of preimage orientation).

Now, for the moduli space $\mathcal{M}_{0, k}\left(\tau_{*} \beta\right)$, we consider

$$
e v_{k, \tau_{*} \beta}^{-1}\left(x_{1} \times \cdots \times x_{k-1}\right) \text {. }
$$

The preimages (2.4) and (2.5) are the same as unoriented sets from the diagram (2.2). Now, there may be several discs in (2.4) but we may compare the signs at the same time because the differences of the signs only depend on the Maslov indices of the maps and the number of marked points, which are fixed in this case.

For the $A$-count, Proposition 2.1 can be used to deduce that (2.4) and (2.5) have the same signed counts if and only if $\mu / 2+k$ is even.

For the case of the $B$-count, the conjugation also changes the cyclic ordering of the marked points to the completely reverse order. This amounts to the new sign contribution $(-1)^{(k-2)(k-1) / 2}$. Hence, for the $B$-count, $(2.4)$ and $(2.5)$ have the same signed counts if $\mu / 2+k+(k-2)(k-1) / 2$ is even.

We provide the following table according to the above criteria, and here we consider the mod 4 dimension of the Lagrangian submanifold and the mod 4 number of point-intersection conditions. If (2.4) and (2.5) have the same orientations, then we denote by 1 and the opposite orientation by -1 . We mark $X$ for the case which does not occur when we consider the condition $(2.3)$.

\begin{tabular}{||c||c|c|c|c||c|c|c|c||}
\hline \multicolumn{1}{||c||}{} & \multicolumn{4}{c||}{$A$ counts } & \multicolumn{4}{c||}{$B$ counts } \\
\hline $\operatorname{dim}(L)$ & $k=0$ & $k=1$ & $k=2$ & $k=3$ & $k=0$ & $k=1$ & $k=2$ & $k=3$ \\
\hline 0 & $X$ & 1 & $X$ & -1 & $X$ & 1 & $X$ & 1 \\
\hline 1 & -1 & 1 & -1 & 1 & 1 & 1 & -1 & -1 \\
\hline 2 & $X$ & 1 & $X$ & -1 & $X$ & 1 & $X$ & 1 \\
\hline 3 & 1 & 1 & 1 & 1 & -1 & 1 & 1 & -1 \\
\hline
\end{tabular}

We only check the first row here, and the other rows can be done similarly. Let $\operatorname{dim}(L)=n=4 i$ for some $i \in \mathbb{N}$, then by (2.3), we have $\mu=4 l k-k+3-4 l$. 
Note that $k$ needs to be an odd number to have $\mu$ an even number. Hence, for the A-count, we have

$$
\mu / 2+k \equiv(k+3) / 2 \quad(\bmod 2)
$$

In the case of $B$-count,

$$
\frac{\mu}{2}+k+\frac{(k-1)(k-2)}{2} \equiv \frac{k^{2}+1}{2}-k \equiv 0 \quad(\bmod 2) .
$$

We interpret the table in the following way. For the case the entry is $(-1)$ (for example $\operatorname{dim}(L) \equiv 4, k \equiv 3(\bmod 4))$, the signed count of real $J$-holomorphic discs (or spheres) adds up to zero, if we count holomorphic dises of both homotopy classes $\beta$ and $\tau_{*} \beta$. For the case the entry is $(+1)$, the signed count of real $J$-holomorphic discs would be twice the signed count of real $J$-holomorphic spheres. Later, we will consider the $A$-count when $\operatorname{dim}(L)=3$ and the $B$ count when $\operatorname{dim}(L)=2$. One can easily notice that in both cases, counts do not cancel out directly by the above table.

\section{Reducible curves and cancelations}

Let $(\Sigma, w)$ be a reducible real $J$-holomorphic disc with boundary on $L$, whose domain $\Sigma$ consists of two disc components, denoted as $\Sigma_{1}, \Sigma_{2}$, which intersect each other at a point. We denote the restriction of the map $w$ on $\Sigma_{i}$ as $w_{i}$ for $i=1,2$. We denote the homology classes as

$$
\beta_{i}:=\left[w_{i}\left(\Sigma_{i}\right)\right] \in H_{2}(M, L),
$$

and we have $\beta=\beta_{1}+\beta_{2}$. The moduli space of such reducible curves form a codimension 1 boundary strata of the moduli space of $J$-holomorphic discs $\mathcal{M}(\beta)$. With the boundary marked points, the moduli space $\mathcal{M}_{0, k}(\beta)$ has codimension 1 boundary strata as

$$
\mathcal{M}_{0, k_{1}+1}\left(\beta_{1}\right)_{e v_{i}} \times_{e v_{0}} \mathcal{M}_{0, k_{2}+1}\left(\beta_{2}\right) .
$$

Now, we consider the conjugate holomorphic discs of the second component of homotopy class $\tau_{*}\left(\beta_{2}\right)$ (See (2.1) for the definition). Then, we consider the fiber product

$$
\mathcal{M}_{0, k_{1}+1}\left(\beta_{1}\right)_{e v_{i}} \times_{e v_{0}} \mathcal{M}_{0, k_{2}+1}\left(\tau_{*}\left(\beta_{2}\right)\right)
$$

This can be considered as a boundary of the moduli space $\mathcal{M}_{0, k}\left(\beta_{1}+\tau_{*}\left(\beta_{2}\right)\right)$. Hence, when we consider

$$
\mathcal{M}_{0, k}\left(\beta_{1}+\beta_{2}\right) \cup \mathcal{M}_{0, k}\left(\beta_{1}+\tau_{*}\left(\beta_{2}\right)\right)
$$

the above two boundary strata disappear by cancellation in mod 2. Similar cancellation occurs for $\mathcal{M}_{0, k}\left(\tau_{*}\left(\beta_{1}\right)+\beta_{2}\right)$ with $\mathcal{M}_{0, k}\left(\tau_{*}\left(\beta_{1}\right)+\tau_{*}\left(\beta_{2}\right)\right)$. Note that there is another choice of matching them for cancelations in mod 2 , namely comparing $\mathcal{M}\left(\beta_{1}+\beta_{2}\right)$ with $\mathcal{M}\left(\tau_{*}\left(\beta_{1}\right)+\beta_{2}\right)$ and $\mathcal{M}\left(\beta_{1}+\tau_{*}\left(\beta_{2}\right)\right)$ with $\mathcal{M}\left(\tau_{*}\left(\beta_{1}\right)+\tau_{*}\left(\beta_{2}\right)\right)$. 
But in either way, if we consider them with orientations, they do not cancel out in general. We proceed more precisely. We have a signed formula as the following.

$$
\begin{gathered}
\mathcal{M}_{0, k_{1}+1}\left(\beta_{1}\right)_{e v_{i}} \times_{e v_{0}} \mathcal{M}_{0, k_{2}+1}\left(\beta_{2}\right)=(-1)^{\epsilon} \partial\left(\mathcal{M}_{0, k}\left(\beta_{1}+\beta_{2}\right)\right), \\
\mathcal{M}_{0, k_{1}+1}\left(\beta_{1}\right)_{e v_{i}} \times_{e v_{0}} \mathcal{M}_{0, k_{2}+1}\left(\tau_{*}\left(\beta_{2}\right)\right)=(-1)^{\epsilon} \partial\left(\mathcal{M}_{0, k}\left(\beta_{1}+\tau_{*}\left(\beta_{2}\right)\right),\right.
\end{gathered}
$$

which has the same $\epsilon$ exponent (see 4.2).

In fact as we compare $\mathcal{M}_{0, k}\left(\beta_{1}+\beta_{2}\right)$ and $\mathcal{M}_{0, k}\left(\beta_{1}+\tau_{*}\left(\beta_{2}\right)\right)$, we need to compare connected components of different cyclic ordering because the conjugation $\tau$ on $\beta_{2}$ will change the cyclic ordering of $\tau_{*} \beta_{2}$ component for $k_{2} \geq 2$. More precisely, for (3.1), suppose the marked points $\left(z_{0}, z_{1}, \ldots, z_{k-1}\right)$ are cyclically ordered and they split into

$$
\left(z_{0}, \ldots, z_{i-1}, z_{*}, z_{i+k_{2}}, \ldots, z_{k-1}\right) \text { and }\left(z_{*}^{\prime}, z_{i}, z_{i+1}, \ldots, z_{i+k_{2}-1}\right) \text {, }
$$

where $*$ denote the marked points where we glue two discs. Let $\sigma$ be the permutation of the index set $\{0, \ldots, k-1\}$ which maps

$$
(0, \ldots, k-1) \mapsto\left(0, \ldots, i-1, i+k_{2}-1, i+k_{2}-2, \ldots, i, i+k_{2}, \ldots, k-1\right) .
$$

Let $\sigma_{2}$ be the permutation of the index set $\left\{*, i, i+1, \ldots, i+k_{2}-1\right)$ which sends

$$
\left(*, i, i+1, \ldots, i+k_{2}-1\right) \mapsto\left(*, i+k_{2}-1, \ldots, i\right) .
$$

Then, we rewrite $(3.1),(3.2)$ as

$$
\mathcal{M}_{0, k_{1}+1}^{\text {main }}\left(\beta_{1}\right)_{e v_{i}} \times_{e v_{0}} \mathcal{M}_{0, k_{2}+1}^{\text {main }}\left(\beta_{2}\right)=(-1)^{\epsilon} \partial\left(\mathcal{M}_{0, k}^{\text {main }}\left(\beta_{1}+\beta_{2}\right)\right),
$$

$$
\mathcal{M}_{0, k_{1}+1}^{\text {main }}\left(\beta_{1}\right)_{e v_{i}} \times_{e v_{0}} \mathcal{M}_{0, k_{2}+1}^{\sigma_{2}(\operatorname{main})}\left(\tau_{*}\left(\beta_{2}\right)\right)=(-1)^{\epsilon} \partial\left(\mathcal{M}_{0, k}^{\sigma(\text { main })}\left(\beta_{1}+\tau_{*}\left(\beta_{2}\right)\right) .\right.
$$

We will see in the next section that if the left hand sides (LHS) of (3.3) and (3.4) cancel out with sign then we may regard that such boundary stratum do not exist. Namely, as we consider cobordisms, as soon as the preimage hits a boundary stratum of a moduli space (death) and there should be a corresponding intersection to the pair (birth) and the cobordism will continue as if there was no boundary in the case that the signs are opposite to each other.

It is easy to see that the cancellation of (3.3) and (3.4) is equivalent to comparing the orientations of $\mathcal{M}_{0, k_{2}+1}^{\text {main }}\left(\beta_{2}\right)$ and $\mathcal{M}_{0, k_{2}+1}^{\sigma_{2}(\text { main })}\left(\tau_{*}\left(\beta_{2}\right)\right)$. Hence for the case of $A$-counts, they do cancel out if and only if

$$
\mu\left(\beta_{2}\right)+2\left(k_{2}+1\right) \equiv 2 \quad(\bmod 4) .
$$

For the case of $B$ counts, the moduli spaces are oriented differently according to the cyclic ordering, hence they do cancel out if and only if

$$
\mu\left(\beta_{2}\right)+2\left(k_{2}+1\right)+k_{2}\left(k_{2}-1\right) \equiv 2 \quad(\bmod 4) .
$$

We remark that clearly there is an analogous story for real $J$-holomorphic spheres as in Figure 1. But usually the reducible curves are not considered as 
Real holomorphic sphere case
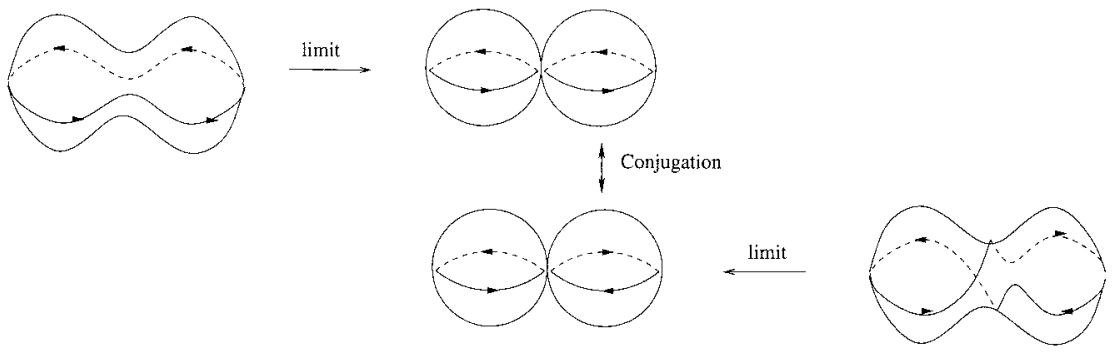

Holomorphic disc case
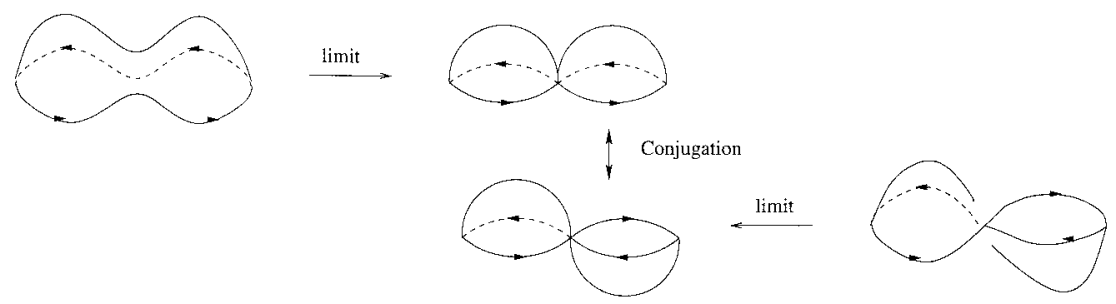

Figure 1. Conjugation

boundary of the moduli spaces of real $J$-holomorphic curves because of this cancellation in mod 2. But the drawback would be that the moduli space of real $J$-holomorphic spheres with marked points seems to be non-orientable in general from the above analysis.

\section{Invariance of $J$-holomorphic disc counts in some cases}

We define a signed count of $J$-holomorphic discs passing through generic real $k$ points and prove its independence with respect to the choice of an almost complex structure or the choice of configuration of real points with the given assumptions.

First, we choose a spherical homology class $\alpha \in H_{2}(M)$, and we say $\beta_{\mathbb{C}}=\alpha$ if there is a holomorphic disc $w$ of class $\beta \in H_{2}(M, L)$ such that its complex double $w_{\mathbb{C}}$ has a homology class $\alpha$. It is not hard to check that if $\beta_{\mathbb{C}}=\alpha$, then $\left(\tau_{*} \beta\right)_{\mathbb{C}}=\alpha$ also.

Let $\boldsymbol{x}=\left(x_{1}, \ldots, x_{k}\right)$ be the configuration of $k$ real points in $L$. Now we define the count of $J$-holomorphic discs for each $\alpha \in H_{2}(M)$ as

$$
I(\alpha, \boldsymbol{x}):=\sharp\left(\bigcup_{\forall \beta, \beta_{\mathbb{C}}=\alpha} e v_{k, \beta}^{-1}\left(x_{1}, \ldots, x_{k}\right)\right),
$$

where $I=A$ or $B$ depending on the orientation of the moduli space of holomorphic dises. 
Theorem 4.1. Let $M, L, J, \tau$ satisfy the assumption given in the beginning of the paper. Then, the $A$-count when $\operatorname{dim}(L)=3$ or the $B$-count when $\operatorname{dim}(L)=$ 2 of $J$-holomorphic discs (hence real $J$-holomorphic spheres) is invariant under the choice of a configuration of real points or the choice of a generic almost complex structure.

Remark 4.1. The case of real configuration of points is proved in Section 5 .

Proof. To prove the independence over the choice of $k$ real points, we take a path

$$
\boldsymbol{x}(t)=\left(x_{1}(t), \ldots, x_{k}(t)\right) \in L^{k}
$$

Consider

$$
\bigcup_{\forall \beta, \beta_{\mathrm{C}=\alpha}} e v_{k, \beta}^{-1}(\boldsymbol{x}(t)) .
$$

By choosing a generic path $\boldsymbol{x}(t)$, we may assume that $\boldsymbol{x}(t)$ is transversal to both the evaluation images of non-singular strata of $\mathcal{M}_{0, k}(\beta)$ and the images of the codimension one strata for all homotopy classes $\beta$ with $\beta_{\mathbb{C}}=\alpha$. Therefore, (4.1) is a collection of one dimensional manifolds with boundaries.

We will prove that the codimension one boundaries resulted from the intersection with reducible curves of (4.1) cancel out, and the remaining boundaries provide the cobordism between $\bigcup_{\forall \beta, \beta_{\mathbb{C}}=\alpha} e v_{k, \beta}^{-1}(\boldsymbol{x}(1))$ and $\bigcup_{\forall \beta, \beta_{\mathbb{C}}=\alpha} e v_{k, \beta}^{-1}(\boldsymbol{x}(0))$, hence proving the invariance of the counting.

Similarly to prove the independence over the choice of a generic almost complex structure, we choose a generic path $\left\{J_{t}\right\}$ (see Section 6), and consider the moduli space $\bigcup_{0 \leq t \leq 1}\{t\} \times \mathcal{M}_{0, k}\left(\beta, J_{t}\right)$. The possible boundaries arise when $t=0$ or $t=1$ or when disc splits into reducible discs at some time $t_{i} \in$ $(0,1)$. Again, it is enough to show that the images of the boundaries of type $e v\left(\left\{t_{i}\right\} \times \partial \mathcal{M}_{0, k}\left(\beta, J_{t_{i}}\right)\right)$ cancel out, to obtain a cobordism between the case of $J_{0}$ and $J_{1}$.

We remark that we do not consider another type of codimension one boundary of the moduli space mentioned in the introduction of this paper. Recall that there may be a strata with a sphere bubble with the main disc component having constant homotopy class. This is of codimension one, not of codimension two, in part because this is not a stable map configuration. But the constant discs cannot intersect $k$-different configuration of points, hence they do not appear in the above cobordism.

We begin the proof of the theorem. First, we have the following lemma which we prove at the end of this section.

Lemma 4.2 (cf. Proposition $23.2[3]$ ).

$$
\partial \mathcal{M}_{0, k}(\beta)=(-1)^{\epsilon} \mathcal{M}_{0, k_{1}+1}\left(\beta_{1}\right)_{e v_{i}} \times_{e v_{0}} \mathcal{M}_{0, k_{2}+1}\left(\beta_{2}\right),
$$

where $\epsilon=\left(k_{1}-1\right)\left(k_{2}-1\right)+n+k_{1}+(i-1)\left(k_{2}+1\right)$. 
Remark 4.2. The actual sign of this formula is not important but only the fact that it is the same for $\beta_{2}$ and $\tau_{*}\left(\beta_{2}\right)$. Hence we postpone its proof to the end of this section.

It is also easy to note that

$$
\partial\left(\{t\} \times \mathcal{M}_{0, k}\left(\beta, J_{t}\right)\right)=(-1)^{\epsilon+1}\{t\} \times\left(\mathcal{M}_{0, k_{1}+1}\left(\beta_{1}\right)_{e v_{i}} \times_{e v_{0}} \mathcal{M}_{0, k_{2}+1}\left(\beta_{2}\right)\right) .
$$

There are evaluation maps at the remaining marked points which we denote as

$$
\begin{gathered}
E V_{\beta_{1}, \beta_{2}}^{k_{1}, k_{2}}: \mathcal{M}_{0, k_{1}+1}\left(\beta_{1}\right)_{e v_{i}} \times_{e v_{0}} \mathcal{M}_{0, k_{2}+1}\left(\beta_{2}\right) \rightarrow L^{k_{1}+k_{2}} \\
E V_{\beta_{1}, \beta_{2}}^{k_{1}, k_{2}, t}:\{t\} \times\left(\mathcal{M}_{0, k_{1}+1}\left(\beta_{1}, J_{t}\right)_{e v_{i}} \times_{e v_{0}} \mathcal{M}_{0, k_{2}+1}\left(\beta_{2}, J_{t}\right)\right) \rightarrow L^{k_{1}+k_{2}} .
\end{gathered}
$$

Now, the boundary contributions resulting from the reducible curves can be written as

$$
\begin{aligned}
& (-1)^{\epsilon}\left(E V_{\beta_{1}, \beta_{2}}^{k_{1}, k_{2}}\right)^{-1}(\boldsymbol{x}(t)), \\
& (-1)^{\epsilon+1}\left(E V_{\beta_{1}, \beta_{2}}^{k_{1}, k_{2}, t}\right)^{-1}(\boldsymbol{x}) .
\end{aligned}
$$

It is easy to see that the difference of the orientations of (4.2) in the case of $\left(\beta_{1}, \beta_{2}\right)$ and the case of $\left(\beta_{1}, \tau_{*} \beta_{2}\right)$, is originated from the difference of orientations in $\mathcal{M}_{k_{2}+1}\left(\beta_{2}\right)$ and $\mathcal{M}_{k_{2}+1}\left(\tau_{*} \beta_{2}\right)$. (Note that we obtain the same (4.2) in each case as unoriented sets.) This can be done exactly as in the previous section. The same is true for (4.3). Hence the cancellation is determined by (3.5) and (3.6)

Therefore we only need to show that these matching pairs of reducible curves are oriented in the opposite way when they contribute to the cobordisms (4.2) or (4.3).

Now, we first observe that to have a nontrivial preimage either in (4.2) or (4.3), the dimension of the moduli space $\mathcal{M}_{0, k_{2}}\left(\beta_{2}\right)$ should at least $k_{2} n-1$. (We restrict the evaluation map to $L^{k_{2}}$, and the image should be at least codimension one to have a non-trivial intersection in both case.) Hence,

$$
\operatorname{dim}\left(\mathcal{M}_{0, k_{2}}\left(\beta_{2}\right)\right)=n+\mu\left(\beta_{2}\right)-3+k_{2} \geq k_{2} n-1 .
$$

Therefore

$$
\mu\left(\beta_{2}\right) \geq k_{2}(n-1)+2-n
$$

Then,

$$
\begin{aligned}
\mu\left(\beta_{1}\right) & =\mu(\beta)-\mu\left(\beta_{2}\right) \\
& \leq k(n-1)+3-n-\left(k_{2}(n-1)+2-n\right) \\
& =k_{1}(n-1)+1 .
\end{aligned}
$$

By applying the same argument starting from $\beta_{1}$, we have obtained the following inequality once we assume the intersection (4.2) or (4.3) is non-trivial:

$$
k_{i}(n-1)+2-n \leq \mu\left(\beta_{i}\right) \leq k_{i}(n-1)+1 \text { for each } i .
$$

Now we consider the cases of $\operatorname{dim}(L)=2$ and $\operatorname{dim}(L)=3$ separately. 
(1) The case $\operatorname{dim}(L)=n=2$. We show that $B$-count is well-defined ( $A$-count is not well-defined in this case).

The above inequality becomes

$$
k_{i} \leq \mu\left(\beta_{i}\right) \leq k_{i}+1 \text {. }
$$

And also we have $\mu(\beta)=k+1$ from (2.3). Note that $k$ must be odd as we assume $L$ is orientable (hence $\mu$ is even). This implies that $k$ splits into an even number, say $k_{1}$ and an odd number, say $k_{2}$. Then, from the above inequality, we have $\mu\left(\beta_{1}\right)=k_{1}$ and $\mu\left(\beta_{2}\right)=k_{2}+1$.

For the proof of the invariance, we show that the evaluation image of $\mathcal{M}_{0, k_{i}+1}(\beta)$ has an opposite orientation as the evaluation image of $\mathcal{M}_{0, k_{i}+1}\left(\tau_{*} \beta\right.$ ) for both $i=0,1$ (In fact, it is enough to show only one of them for the proof).

More precisely, the change of sign due to the Proposition 2.1 plus the sign from the cyclic ordering permutation is as follows. For even $k_{1}$,

$$
k_{1}+2\left(k_{1}+1\right)+\left(k_{1}-1\right) k_{1} \equiv k_{1}^{2}+2 \quad(\bmod 4) .
$$

But $k_{1}^{2}$ is always a multiple of 4 , hence from the proposition, it is orientation reversing. For odd $k_{2}$,

$$
k_{2}+1+2\left(k_{2}+1\right)+\left(k_{2}-1\right) k_{2} \equiv k_{2}^{2}+1 \quad(\bmod 4) .
$$

But $k_{1}^{2}$ is always of type $4 j+1$, hence from the proposition, it is orientation reversing. This proves the invariance of counting for $\operatorname{dim}(L)=2$.

(2) The case $\operatorname{dim}(L)=n=3$. In this case we show that $A$-count is welldefined. From the inequality (4.5), we have

$$
2 k_{i}-1 \leq \mu\left(\beta_{i}\right) \leq 2 k_{i}+1 .
$$

But, since $\mu\left(\beta_{i}\right)$ is an even number, we have $\mu\left(\beta_{i}\right)=2 k_{i}$ for $i=1,2$. It is enough to show that we have $\mu\left(\beta_{i}\right) / 2+k_{i}+1$ is odd. But, it is obvious since $\mu\left(\beta_{i}\right) / 2+k_{i}+1=2 k_{i}+1$ is always odd. This finishes the proof of invariance for the case $\operatorname{dim}(L)=3$.

Proof. This is the proof of the Lemma 4.2. We modify the result given in Proposition 23.2 [3] which can be stated as

$$
\partial \mathcal{M}_{0, k}(\beta)=(-1)^{\left(k_{1}-1\right)\left(k_{2}-1\right)+n+k_{1}} \mathcal{M}_{0, k_{1}+1}\left(\beta_{1}\right)_{e v_{1}} \times_{e v_{0}} \mathcal{M}_{0, k_{2}+1}\left(\beta_{2}\right) .
$$

The only difference here from our lemma is that the $\beta_{2}$ disc intersect $\beta_{1}$ disc at the first marked point, where as in our case it intersects at the $i$-th marked point. (We assume the readers are familiar to the Proposition 23.2 for this proof) To remedy it, We first reorder the orientation of the marked points of $\mathcal{M}_{0, k}(\beta)$ as

$$
\left(\partial D_{0}^{2}\right) \cdots\left(\partial D_{k-1}^{2}\right) \mapsto\left(\partial D_{i-1}^{2}\right) \cdots\left(\partial D_{k-1}^{2}\right)\left(\partial D_{0}^{2}\right) \cdots\left(\partial D_{i-2}^{2}\right),
$$


where $\partial D_{i}^{2}$ denotes the $i$-th marked point for $i=0, \ldots, k-1$. This provides a sign $(-1)^{(i-k+1)(i-1)}$. Now, we apply the proposition which now splits at $i$-th marked point of $\beta_{1}$ disc. Then, the ordering of the marked points of the resulting space $\mathcal{M}_{0, k_{1}+1}\left(\beta_{1}\right)$ is

$$
\left(\partial D_{i-1}^{2}\right)\left(\partial D_{i}^{2}\right)\left(\partial D_{i+k_{2}}^{2}\right) \cdots\left(\partial D_{i-2}^{2}\right) \text {. }
$$

To return to the original order of marked points, we need additional sign

$$
(-1)^{(i-1)\left(2+k-i-k_{2}+2\right)} \text {. }
$$

Hence by summing up the two sign factors, we prove the lemma.

\section{The case of real configuration of points}

The main invariance theorem can be extended to the case of real configuration of points. Let $\boldsymbol{x}=\left\{x_{1}, \ldots, x_{k}\right\}$ be the configuration of $k$ points on the symplectic manifold $M$. We call $\boldsymbol{x}$ is the real configuration of $k$ points if

$$
\left\{\tau\left(x_{1}\right), \ldots, \tau\left(x_{k}\right)\right\}=\left\{x_{1}, \ldots, x_{k}\right\} .
$$

We denote by $r$ the number of real points $x_{j}$ with $\tau\left(x_{j}\right)=x_{j}$, and denote by $2 c=k-r$. Then, there are $c$ conjugate pairs in this real configuration of points. Now, we assume without loss of generality that the real configuration is arranged so that $\left(x_{1}, x_{2}\right), \ldots,\left(x_{2 c-1}, x_{2 c}\right)$ form conjugate pairs. Then, for any such real configuration $\boldsymbol{x}$, we consider

$X(x):=\left\{\left(t_{1}, \ldots, t_{c}, x_{2 c+1}, \ldots, x_{k}\right) \mid t_{j}=x_{2 j}\right.$ or $t_{j}=x_{2 j-1}=\tau\left(x_{2 j}\right)$ for $\left.1 \leq j \leq c\right\}$.

We define a count of $J$-holomorphic discs which intersect $r$ real points at the boundary and intersect either of $c$ conjugate pairs at the interior. Consider the evaluation map $E v_{(c, r, \beta)}: \mathcal{M}_{c, r}(\beta) \rightarrow M^{c} \times L^{r}$ defined by,

$$
\left(u, z_{0}^{+}, \ldots, z_{c-1}^{+}, z_{0}, \ldots, z_{r-1}\right) \mapsto\left(u\left(z_{0}^{+}\right), \ldots, u\left(z_{c-1}^{+}\right), u\left(z_{0}\right), \ldots, u\left(z_{r-1}\right)\right) .
$$

Then, we define the signed counting as

$$
I(\alpha, \boldsymbol{x})=\sharp\left(\bigcup_{\forall \beta, \beta_{\mathbb{C}}=\alpha}\left(E v_{(c, r, \beta)}\right)^{-1}(X(\boldsymbol{x})),\right.
$$

where $I=A$ or $B$ depending on the sign of the moduli spaces. It is not hard to check that such a count is finite if the equation (2.3) holds.

Proposition 5.1. Theorem 4.1 holds also for the real configuration of points.

Proof. To compare the change of an orientation under the conjugation, we consider the following commuting diagram, which is a modified version of (2.2).

$$
\begin{array}{ccc}
\mathcal{M}_{c, r}(\beta) & \stackrel{E v_{(c, r, \beta)}}{\longrightarrow} & M^{c} \times L^{r} \\
\tau_{*} \downarrow & & \left(\tau_{c}, i d\right) \downarrow \\
\mathcal{M}_{c, r}\left(\tau_{*}(\beta)\right) & \stackrel{\left.E v_{(c, r, r}, \beta\right)}{\longrightarrow} & M^{c} \times L^{r}
\end{array}
$$


Here $\tau_{c}: M^{c} \rightarrow M^{c}$ defined by $\left(x_{1}, \ldots, x_{c}\right) \mapsto\left(\tau\left(x_{1}\right), \ldots, \tau\left(x_{c}\right)\right)$ and $\tau_{*}\left(w, z_{0}^{+}, \ldots, z_{c-1}^{+}, z_{0}, \ldots, z_{r-1}\right)=\left(\widetilde{w}, \overline{z_{0}^{+}}, \ldots, \overline{z_{c-1}^{+}}, \overline{z_{0}}, \ldots, \overline{z_{r-1}}\right)$.

Lemma 5.2. The map $\tau_{*}$ is orientation preserving if and only if $\mu(\beta)+2 c_{1}+$ $2 r \equiv 0(\bmod 4)$. And the map $\tau_{c}$ is orientation preserving if and only if $c$. $\operatorname{dim}(M) \equiv 0(\bmod 4)$.

The proof of the lemma follows from the Proposition 2.1 with the fact that conjugation $\mathbb{C}^{c} \rightarrow \mathbb{C}^{c}$ is orientation preserving if and only if $c$ is even.

It is easy to see that there is a bijection between the sets $\left(E v_{(c, r, \beta)}\right)^{-1}(X(x))$ and $\left(E v_{\left(c, r, \tau_{*} \beta\right)}\right)^{-1}(X(x))$. And we compare their orientations by considering the orientation changes under both maps, $\tau_{*}$ and $\left(\tau_{c}, i d\right)$ together from the diagram 5.1.

In the case of $A$-count with $\operatorname{dim}(L)=3$, the both inverse images carry the same orientations since

$$
\mu(\beta)+2 c+2 r+6 c=\mu(\beta)+2 k \equiv 0 \quad(\bmod 4) .
$$

The last line follows from the table in Section 2.3. In the case of $B$-count with $\operatorname{dim}(L)=2$, the both inverse image carry the same orientation as

$$
\mu(\beta)+2 c+2 r+0+(r-2)(r-1) \equiv r^{2}+3 \equiv 0 \quad(\bmod 4) .
$$

Here we use the fact that $\mu(\beta)=k+1$ for $\operatorname{dim}(L)=2$.

Hence the same table as in the Section 2.3 works for these cases, which shows that the contribution from the conjugating discs do not cancel out in these cases.

Now, we consider the proof of the invariance of the counting. We first show that the we obtain the same inequality (4.5) for the case relevant to the proof. Suppose in the limit we have a stable disc with the domain consists of two disc components $\Sigma_{1}$ and $\Sigma_{2}$. We assume that each component has $c_{i}$ interior marked points and $r_{i}$ boundary marked points. we consider $E v_{\left(c_{2}, r_{2}, \beta_{2}\right)}: \mathcal{M}_{c_{2}, r_{2}}(\beta) \rightarrow$ $M^{c_{2}} \times L^{r_{2}}$. (The case we vary an almost complex structure $J_{t}$ can be done analogously and we omit the proof in that case). Now, we observe as before that this stable limit curve will contribute to the counting if the image of $E v_{\left(c_{2}, r_{2}, \beta_{2}\right)}$ is at most of codimension one. Hence, we have

$$
n+\mu\left(\beta_{2}\right)-3+2 c_{2}+r_{2} \geq c_{2} \cdot 2 n+r_{2} \cdot n-1=k_{2} n-1 .
$$

Note that this is the same inequality as (4.4), hence we obtain the same inequality (4.5).

Now we can show the cancellation of the image of reducible curves as in the proof of the main theorem. We compare the sign of $E v_{\left(c_{i}, r_{i}+1, \beta_{i}\right)}$ and $E v_{\left(c_{i}, r_{i}+1, \tau_{*} \beta_{i}\right)}$ upon the condition (4.5). First, we consider the case $\operatorname{dim}(L)=$ 2. We may assume without loss of generality that $k_{1}$ is even and $k_{2}$ is odd and $\mu\left(\beta_{1}\right)=k_{1}$ and $\mu\left(\beta_{2}\right)=k_{2}+1$. The fact that they have the opposite orientations follow from

$$
\mu\left(\beta_{1}\right)+2 c_{1}+2\left(r_{1}+1\right)+r_{1}\left(r_{1}-1\right) \equiv r_{1}^{2}+2 r_{1}+2 \equiv 2 \quad(\bmod 4),
$$




$$
\mu\left(\beta_{2}\right)+2 c_{2}+2\left(r_{2}+1\right)+r_{2}\left(r_{2}-1\right) \equiv r_{2}^{2}+2 r_{2}+3 \equiv 2 \quad(\bmod 4),
$$

where the last equalities follow because $r_{1}$ is even and $r_{2}$ is odd.

For the case $\operatorname{dim}(L)=3$, they still have the opposite orientations because

$$
\mu\left(\beta_{i}\right)+2 c_{i}+2\left(r_{i}+1\right)+2 c_{i} \equiv 2 k_{i}+4 c_{i}+2 r_{i}+2 \equiv 4 k_{i}+2 \equiv 2 \quad(\bmod 4)
$$

Hence we obtain the main theorem for the real configuration of points.

\section{Remark about the moduli spaces}

Here, we state rather well-known facts about moduli space of real $J$-holomorphic spheres or dises.

Recall from [7] that a $k$-dimensional pseudo-cycle in $M$ is a smooth map $f: V \rightarrow M$ defined on an oriented $k$-dimensional manifold $V$ such that the $\operatorname{dim}\left(\Omega_{f}\right) \leq \operatorname{dim}(V)-2$, where $\Omega_{f}=\bigcap_{K \subset V, K c p t} \overline{f(V-K)}$. Let $W$ be an oriented $k$-dimensional manifold $W$ with codimension one boundary, then we will call a smooth map $F: W \rightarrow M$ a pseudo-chain if $\operatorname{dim}\left(\Omega_{F}\right) \leq k-2$. For $W$ a certain moduli space of holomorphic discs, we say that a pseudo-chain $f: W \rightarrow M$ defines a pseudo-cycle modulo disc bubbling if all the codimension one boundary $\partial W$ occur due to disc bubbling phenomenons on the moduli space $W$.

We denote by $\mathbb{R} \mathcal{M}(M, \alpha)^{*}$ the moduli space of simple real $J$-holomorphic spheres which preserve real structures. Namely, $u:\left(S^{2}\right.$, conj $) \rightarrow(M, \tau)$ satisfies

$$
u(\operatorname{conj}(z))=\tau(u(z)) .
$$

Here $\alpha \in H_{2}(M)$ is a spherical homology class. Recall that in [7], it was shown that the moduli space of simple $J$-holomorphic spheres form a pseudo-cycle for a generic $J$ in the strongly semi-positive case. In the case of simple real $J$-holomorphic spheres, the similar results holds in the following way. First, as shown in [10] the linearized $\bar{\partial}$ operator, $D$ is $\mathbb{Z} / 2 \mathbb{Z}$-equivariant, where the actions are given by the involutions. Hence for a generic $J$ with $\tau_{*} J=-J$, one can show that the moduli space of simple real non-singular $J$-holomorphic spheres form a smooth manifold by considering the equivariant version of the proof given in [7]. Moreover, in the case of real $J$-holomorphic curves, Gromov-compactness, and gluing of the two real $J$-holomorphic curves work as in the case of [7]. This is because the Lemma 6.1.2 of [7] can be proved $\mathbb{Z} / 2 \mathbb{Z}$-equivariantly as $D$ and $u$ are $\mathbb{Z} / 2 \mathbb{Z}$-equivariant. The lemma states that for the space of compatible almost complex structures $\mathcal{J}$, the evaluation map $e v_{z}: \mathcal{M}(A, \mathcal{J})^{*} \rightarrow M$ for a fixed $z \in C P^{1}$ is submersive, and it was crucially used to show that the moduli spaces form a pseudo-cycle, and to make the gluing of two $J$-holomorphic curves work.

Hence, everything mentioned above also works equally well for $\mathcal{M}(\beta)$, the moduli space of $\tau$-simple $J$-holomorphic discs. Here we call $J$-holomorphic disc $w, \tau$-simple if $w$ is injective away from a discrete set of points in the interior of $D^{2}$, and additionally, its complex double $w_{\mathbb{C}}$ is a simple $J$-holomorphic sphere. 
( $w$ is injective at $p$ if $d u(p) \neq 0$ and $u^{-1}(u(p))=\{p\}$ ). This is the moduli space which is used throughout the paper.

It can be shown that non-simple real $J$-holomorphic spheres have images of codimension two or higher, either by using the structure theorem of real holomorphic discs, or by using the general structure theorem of pseudo-holomorphic discs by Kwon and Oh [6]. Hence for the intersection theory, we may consider only $\tau$-simple holomorphic discs.

\section{References}

[1] C.-H. Cho, Holomorphic discs, spin structures, and Floer cohomology of the Clifford torus, Int. Math. Res. Not. (2004), no. 35, 1803-1843.

[2] _ Counting real J-holomorphic discs and spheres in dimension four and six, preprint: arXiv:math.SG/0604501.

[3] K. Fukaya, Y.-G. Oh, H. Ohta, and K. Ono, Lagrangian Intersection Floer Theory Anomaly and Obstruction, 2000, Preprint.

[4] V. Guillemin and A. Pollack, Differential Topology, Prentice-Hall, Inc., Englewood Cliffs, N.J., 1974.

[5] S.-C. Kwon, Transversality properties on the moduli space of genus 0 stable maps to a smooth rational projective surface and their real enumerative implications, preprint: arXiv:math.AG/0410379.

[6] D.-S. Kwon and Y.-G. Oh, Structure of the image of (pseudo)-holomorphic discs with totally real boundary condition, Appendix 1 by Jean-Pierre Rosay, Comm. Anal. Geom. $8(2000)$, no. $1,31-82$.

[7] D. McDuff and D. Salamon, J-holomorphic Curves and Quantum Cohomology, University Lecture Series, 6. American Mathematical Society, Providence, RI, 1994.

[8] J. Solomon, Intersection theory on the moduli space of holomorphic curves with Lagrangian boundary conditions, Preprint: arXiv:math.SG/0606429.

[9] O. Viro, introduction to topology of real algebraic varieties, article available at http://www.math.uu.se/ oleg/es/index.html.

[10] J.-Y. Welschinger, Invariants of real symplectic 4-manifolds and lower bounds in real enumerative geometry, Invent. Math. 162 (2005), no. 1, 195-234.

[11] Spinor states of real rational curves in real algebraic convex 3-manifolds and enumerative invariants, Duke Math. J. 127 (2005), no. 1, 89-121.

[12] —_, Enumerative invariants of strongly semipositive real symplectic manifolds, preprint: arXiv:math.AG/0509121.

Department of Mathematical Sciences

Seoul National University

SEOUL 151-747, KorEA

E-mail address: chocheolesnu.ac.kr 